\title{
An Epidemiological Survey of Childhood Developmental Disorders in primary school children
}

\author{
Vishakha Shinde ${ }^{1}$ \\ Jill Soni ${ }^{2}$ \\ Rajshree Naik ${ }^{3}$ \\ ${ }^{1}$ Assistant Professor \\ ${ }^{2}$ Intern \\ ${ }^{3}$ Professor and Head \\ Department of Physiotherapy, Lokmanya Tilak Municipal Medical College, Mumbai \\ E-mail-vishakhaphysio271@gmail.com
}

\section{ABSTRACT}

Developmental disorders are common in childhood and are seen in primary school children. Early detection shall lead to early intervention and better outcomes at a later age. The following study was aimed to assess the prevalence of developmental disorders in a school based sample of primary school children. 400 children from a school were screened using the Strengths and Difficulties questionnaire where the teacher was the main informant. Out of the 400 children screened, hyperactivity and emotional symptoms were the most common disorders noted. Many students were in the borderline range and would need assessment. The disorders showed a grave impact on learning and their school performance. The study however was superficial and did not look at family and personal factors. The study indicated that developmental disorders are common in primary school children and need assessment and early intervention to prevent further progression.

Key words: developmental disorders, school children, primary school, conduct problems.

\section{INTRODUCTION}

"Developmental disorder" and "developmental disability" refer to a childhood mental or physical impairment or combination of mental and physical impairments that result in substantial functional limitations in major life activities. Childhood disorders, often labelled as developmental disorders or learning disorders, most often occur and are diagnosed when the child is of school-age [1]. Although some adults may also relate to some of the symptoms of these disorders, typically the disorder's symptoms need to have first appeared at some point in the person's childhood [2]. Current detection rates of developmental disorders are lower than their actual prevalence, which suggests that the challenges to early identification of children with developmental disorders have not been overcome. Early identification should lead to further evaluation, diagnosis, and treatment [3]. Early intervention is available for a wide range of developmental disorders; their prompt identification can spur specific and 
appropriate therapeutic interventions [4]. Childhood developmental disorders tend to show their first symptoms during childhood. Current detection rates of developmental disorders are lower than their actual prevalence, which suggests that the challenges to early identification of children with developmental disorders have not been overcome. Early intervention is available for a wide range of developmental disorders; their prompt identification can spur specific and appropriate therapeutic interventions. To address a large group of children, one must know the extent of the problem in the community. The aim of the current study was to conduct an epidemiological survey of developmental disorders in primary school children studying in urban schools in Mumbai.

\section{METHODOLOGY}

The study was an observational multicentre study. The study was school based and non sponsored. The sample was 400 children studying in primary schools across Mumbai. The inclusion criteria were primary school children studying in the school and attending school regularly. Parental consent was obtained and school permission was taken for the study. There were three parameters that were assessed - the difficulty score, the prosocial score and the impact score. The scale used in the assessment was the Strengths and Difficulties Questionnaire (SDQ) [5]. The SDQ is a brief questionnaire that can be administered to the parents and teachers of 4-16 year olds [6]. Besides covering common areas of emotional and behavioural difficulties, it also enquires whether the informant thinks that the child has a problem in these areas and, if so, asks about resultant distress and social impairment. Computerised algorithms exist for predicting psychiatric disorder by bringing together information on symptoms and impact from SDQs completed by multiple informants [7] The algorithm makes separate predictions for three groups of disorders, namely conduct-oppositional disorders, hyperactivity-inattention disorders, and anxiety-depressive disorders. Each is predicted to be unlikely, possible or probable. Predictions of these three groups of disorders are combined to generate an overall prediction about the presence or absence of any psychiatric disorder.

Systematic random sampling was used in the study. All schools will be listed. One school will be selected using the lottery method using simple random sampling. All students in the given age group will be listed and then chosen according to the sampling interval found. First student will be randomly selected and others henceforth based on sampling interval using systematic sampling until sample size is attained.

Sample size was calculated using the formula $n=4 \times P \times Q / d 2$

where

$\mathrm{P}=$ prevalence of childhood developmental disorder in the population found by previous studies. $\mathrm{Q}=100-\mathrm{P}$ and $\mathrm{d}=$ confidence interval $(\mathrm{d}=5$ at $99 \%$ confidence interval)

\section{RESULTS}

Based on the results of the SDQ scores it was observed that that out of 400 children screened, $5 \%(n=20)$ showed emotional symptoms while $7 \%(n=28)$ showed conduct features and as high as $10 \%(n=40)$ were borderline in this regard. $6 \%$ $(n=24)$ of the sample tested positive for hyperactivity. $7 \%(n=28)$ children reported peer problems as a main factor. On assessing the overall difficulties score it was noted that $9 \%$ had abnormal scores $(n=36)$ while $13 \%$ were borderline in this arena $(n=52)$. On the prosocial scale, $12 \%(n=48)$ were abnormal while $13 \%(n=52)$ were borderline. 
$46 \%$ of the borderline children were impacted by their scores while $58 \%$ of children in the abnormal range were impacted. The children displaying conduct difficulties and prosocial behavior problems are shown to have the highest impact on their classroom learning and peer relations. Out of the total population, $17 \%$ showed conduct problems, $16 \%$ peer problems, $13 \%$ hyperactivity, $9 \%$ emotional disturbances and $25 \%$ prosocial affection. Childhood developmental disorder accounting for $22.1 \%$ of those affected. Out of borderline children noted, $25 \%$ showed quite a lot impact and $21 \%$ a great deal of impact on classroom learning and peer relations. Out of abnormal children noted, a whooping $38 \%$ showed quite a lot impact, whereas $19 \%$ were seen to have a great deal of impact. Out of the domains contributing to the total difficulty score, hyperactivity showed to have the highest impact

\section{DISCUSSION}

It is emerging convincingly that the developmental disorders tend to show significant symptoms in children between the age group of 4-10years. There has been a significant prevalence of such childhood developmental disorders in the world. Studies emerging from different parts of India also indicate similar trends. Among the total difficulties noted, conduct problems was the most affected domain. They showed traits of often losing temper, cheating or fighting with other children with occasional stealing and lying. Environmental factors like contributing maybe child abuse, dysfunctional family or unsupportive residing environments which were not assessed in our paper. Among the hyperactivity problem noted it showed lack of attention, restlessness and impulsiveness which affected his peer relations too. This has been attributed to hyperthyroidism, premature births, brain damage in womb or early life years or drinking alcohol and smoking while pregnant. These factors were not assessed in the study.

In the present study, childhood developmental disorder was found to be $22.1 \%$. A similar finding was reported from another study in India that showed childhood developmental disorder of $36.30 \%$ from various school based studies. Another similar study showed $23.33 \%$ of prevalence of such disorders through school based meta analytical studies [8]. Our study showed among the various childhood developmental disorders noted, conduct problems were the highest affecting $17 \%$ of the kids. The probable causes could be as defined earlier. The highest impact is seen in kids with hyperactivity and prosocial behavior problems affecting their classroom learning and peer relations. The strength of our study remains that it provides an overview of burden of childhood developmental disorder among representative sample of primary school students for the first time and can prove to be a benchmark for future comparisons by public health personnel. It also indicates the need for early detection both at home or school, timely intervention and devise meaningful outcome measures, further research on their early detection tools for childhood developmental disorders and also to monitor the trend in near future [9].

The limitation of our study was that teachers rather than parents were chosen as informants and this may have had a bias element for our study. The effect of gender was not studied though the school was co-education and the impact of home and family life as well as other pertinent factors were not studied.

\section{REFERENCES}


1. Bhola P, Kapur M. Child and Adolescent Psychiatric Epidemiology in India. Indian J Psychiatry 2003;45(4):208-17.

2. Achenbach TM, McConaughy SH, Howell CT. Child/adolescent behavioural and emotional problems: implications of cross-informant correlations for situational specificity. Psychol Bull 1987; 101(2):213-32.

3. Goodman R, Ford T, Simmons H, Gatward R, Meltzer H. Using the Strengths and Difficulties Questionnaire (SDQ) to screen for child psychiatric disorders in a community sample. Br J Psychiatry 2000;177(6):534-9.

4. Goodman R, Renfrew $D$, Mullick M. Predicting type of psychiatric disorder from Strengths and Difficulties Questionnaire (SDQ) scores in child mental health clinics in London and Dhaka. Eur Child Adolesc Psychiatry 2000;9(2):129-34.

5. Goodman R. The Strengths and Difficulties Questionnaire: a research note. J Child Psychol Psychiatry 1997;38(5):581-6.

6. Goodman R. Psychometric properties of the strengths and difficulties questionnaire. J Am Acad Child Adolesc Psychiatry 2001;40(11):1337-45.

7. Goodman R, Scott S. Comparing the Strengths and Difficulties Questionnaire and the Child Behavior Checklist: is small beautiful ? J Abnorm Child Psychol 1999;27(1):17-24.

8. Srinath S, Girimaji SC, Gururaj G, Seshadri S, Subbakrishna DK, Bhola P, Kumar N. Epidemiological study of child \& adolescent psychiatric disorders in urban \& rural areas of Bangalore, India. Ind J Med Res 2005;122(1):67-73.

9. Belfer ML. Child and adolescent mental disorders: the magnitude of the problem across the globe. J Child Psychol Psychiatry 2008;49(3):226-36.

Acknowledgements - Nil

Conflict of Interest - Nil

Funding - Nil. 